\title{
The Effect of Acid Treatment on the Black Silicon for Solar Cells
}

\author{
Yongtao $\mathrm{Li}^{1}$, a , BangWu Liü,b \\ ${ }^{1}$ Key Laboratory of Microelectronics Devices \& Integrated Technology, Institute of Microelectronics, \\ Chinese Academy of Sciences, Beijing 100029, China \\ ${ }^{2}$ Shandong Xindaneng Environmental Protection Technology Co. Ltd., 766\#, Lanzhou road, Heze \\ high-tech developmental zone, Shandong 274000, China \\ aliyongtao@ime.ac.cn, ${ }^{\mathrm{b}}$ liubangwu@ime.ac.cn
}

Keywords: black silicon; acid treatment; solar cell.

Abstract. The HNO3/HF solutions etching has been used to improve the properties of the black silicon for solar cells. The effect of the HNO3/HF ratio and etching time on the black silicon has been investigated by scanning electron microscope, UV-VIS-NIR spectrophotometer, external quantum efficiency and I-V test system. Results show that the HNO3-rich HF-HNO3 solution etching can optimize the structure and remove the damage of the black silicon. After acid etching, the efficiency of the black silicon solar cell can be improved by $1 \%$.

\section{Introduction}

Optical losses caused by high reflectance of natural silicon wafer are critical to limit the conversion efficiency of crystalline silicon solar cells. Reducing surface reflectance to enhance light absorption is very important to improve the conversion efficiency of crystalline silicon solar cell. The efficiency of the multicrystalline silicon solar cells is about $1 \sim 2 \%$ lower than that of the single crystalline cells. One of the most important reasons for this difference is the lack of an effective method to texture mc-Si. Isotropic etching in acid solution is widely used in industry [1]. However, the reflectance of acid textured wafer is still as high as $23 \%$, which limits its applications for high-efficient solar cells. To further reduce the reflectance, types of approaches have been reported, such as electrochemical etching[2], reactive ion etching[3-6], laser texturing by femtosecond pulses[7,8] and metal-induced chemical etching [9-11]. In our previous work, plasma immersion ion implantation (PIII) has been put forward to produce black silicon to texture the silicon surface [12-14]. Although the reflectance can be reduced to $0.5 \%$, the efficiency of the solar cell cannot be improved due to the damage induced by plasma.

In the present study, acid treatment is used to remove the damage to improve the efficiency of the black silicon solar cells. The effect of the acid treatment on the microstructure black silicon and the properties of the solar cells will be investigated in detail.

\section{Experiments}

The material used for experiments was commercially available boron-doped p-type multicrystalline silicon wafers obtained from the ingot by wire sawing with thickness $\sim 200 \mu \mathrm{m}$, area $156 \mathrm{~mm} \times 156$ $\mathrm{mm}$, and resistivity $1 \sim 3 \Omega \mathrm{cm}$. Damage on the surface induced by wire cutting was removed by etching in $10 \% \mathrm{NaOH}$ solution at $80^{\circ} \mathrm{C}$. The black silicon was prepared by plasma immersion ion implantation process on home-made equipment and subsequently subjected to acid etching in $\mathrm{HF} / \mathrm{HNO} 3$ solutions. After that, the black silicon wafers were phosphorus doped using phosphorous oxychloride ( $\mathrm{POCl} 3)$ as the dopant source and then subjected to edge etching through reactive ion etching and removing phosphosilicate glass (PSG) layer with diluted HF. Silicon-nitride layer for passivation was grown by plasma enhanced chemical vapor deposition (PECVD) process. Finally, the front and back metallization of all the wafers were carried out by screen-printing technique and followed by baking and co-firing in a conveyer belt furnace. 
The microstructure of the black silicon before and after the acid etching was characterized by scanning electron microscope (SEM). The surface reflectance was examined by a UV-VIS-NIR spectrophotometer (Varian Cary 5000) equipped with an integrating sphere detector in the wavelength from 300 to $1100 \mathrm{~nm}$. The internal quantum efficiency (IQE) was measured on Solar Cell Scan 100 quantum efficiency measurement system. I-V characterization was used to evaluate the black silicon solar cell under one sun global solar spectrum of AM 1.5 at $25^{\circ} \mathrm{C}$.

\section{Results and discussion}

\section{Effect of $\mathrm{HNO}_{3} / \mathrm{HF}$ ratio on the microstructure of black silicon}

The microstructure of the black silicon before and after acid etching is presented in Figure 1. The surface of the black silicon produced by plasma immersion ion implantation appears porous-sponge like structure. However the porous structure is not uniform and smooth, which will deteriorate the passivation effect of SiNx film, furthermore reduce the efficiency of the black silicon solar cells. After HF-rich HF-HNO3 solution etching, the microstructure of the black silicon becomes worse and more nanoscale pore appears. The HF-rich HF-HNO3 solution can result in rough surface of the silicon and porous silicon (PS) may be formed. After HNO3-rich HF-HNO3 solution etching, the microstructure of the black silicon becomes smooth. With the HNO3/ HF ratio increasing, the microstructure of the black silicon becomes more smooth and uniform. When the HNO3/HF ratio is 25 , honeycomb structure appears, which is an ideal structure for solar cells application. The structure evolution mechanism of the black silicon is shown in Figure 2. The etching of black silicon in the HF-HNO3 system follows a chemical process with two basic reaction steps. In the first step, silicon is oxidized by HNO3 which is followed by dissolution of the oxidized Si by HF. The overall reaction is [15]:

$$
3 \mathrm{Si}+4 \mathrm{HNO}+18 \mathrm{HF} \leftrightarrow 3 \mathrm{H} 2 \mathrm{SiF} 6+4 \mathrm{NO}+8 \mathrm{H} 2 \mathrm{O}
$$

Different $\mathrm{HNO} / \mathrm{HF}$ ratio will decide different reaction mechanism, resulting in different microstructures. During the HNO3-rich HF-HNO3 solution etching reactions, the minute structure will be smoothed first.
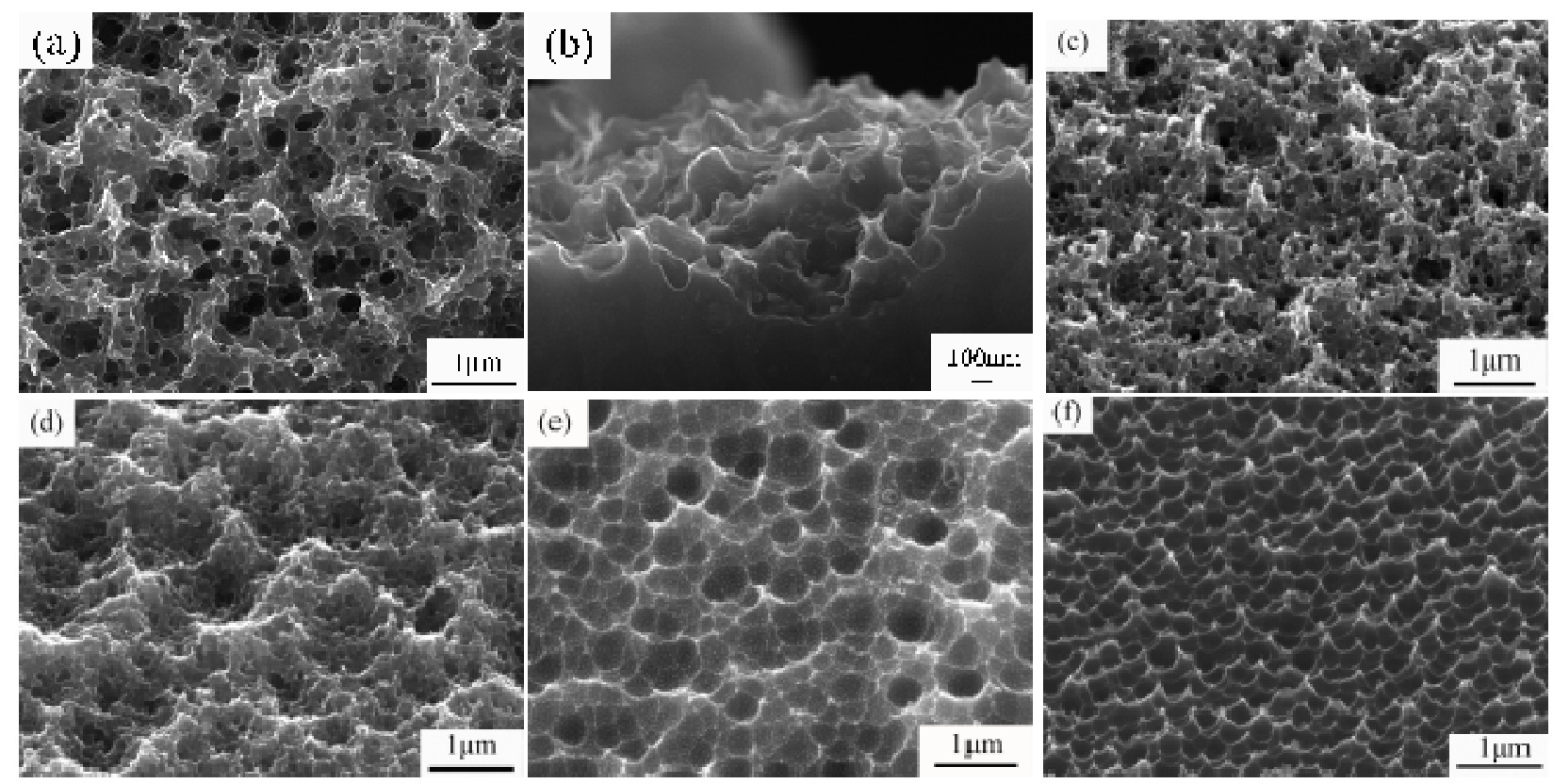

Fig. 1 The microstructure of black silicon before and after acid etching

(a)before etching (top view); (b) before etching (side view); (c) etching by HNO3/HF with a ratio of $1 / 4$; (d) etching by HNO3/HF with a ratio of $1 / 1$; (e) etching by HNO3/HF with a ratio of $4 / 1$; (f) etching by $\mathrm{HNO} / \mathrm{HF}$ with a ratio of $25 / 1$ 

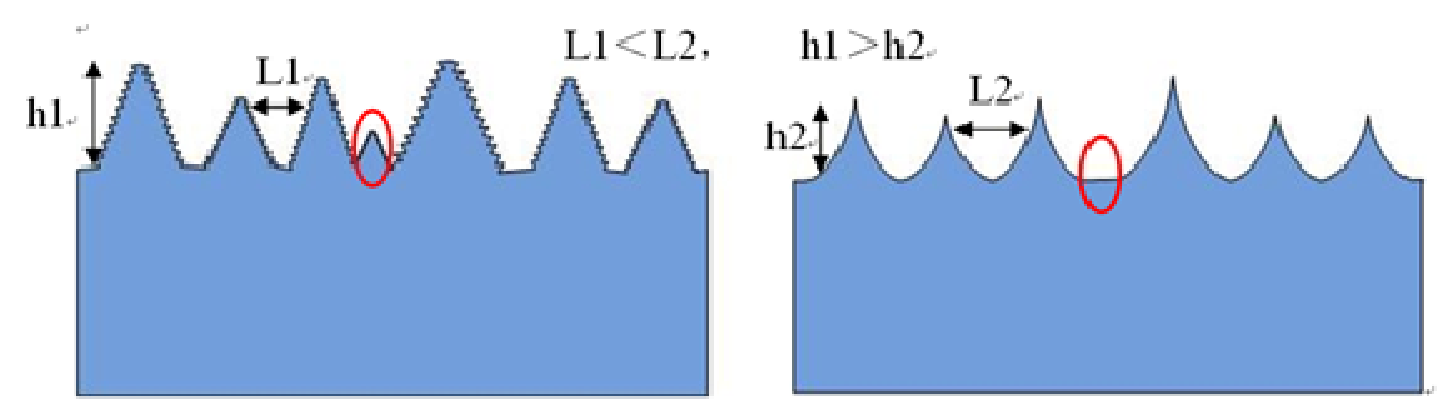

Fig. 2 The structure evolution mechanism of the black silicon by acid etching

\section{Effect of the etching time on the black silicon}

Keeping the $\mathrm{HF} / \mathrm{HNO} 3 / \mathrm{H} 2 \mathrm{O}$ ratio at $1 / 25 / 18$, the microstructure of the black silicon varying with the etching time is presented in figure 3. It can be seen that the microstructure of the black silicon changes from the porous-sponge like structure to needle like to honeycomb structure with increasing the etching time. Also, with increasing the etching time, the structure of the black silicon becomes shallow, which will increase the reflectance of the black silicon, as presented in figure 4.
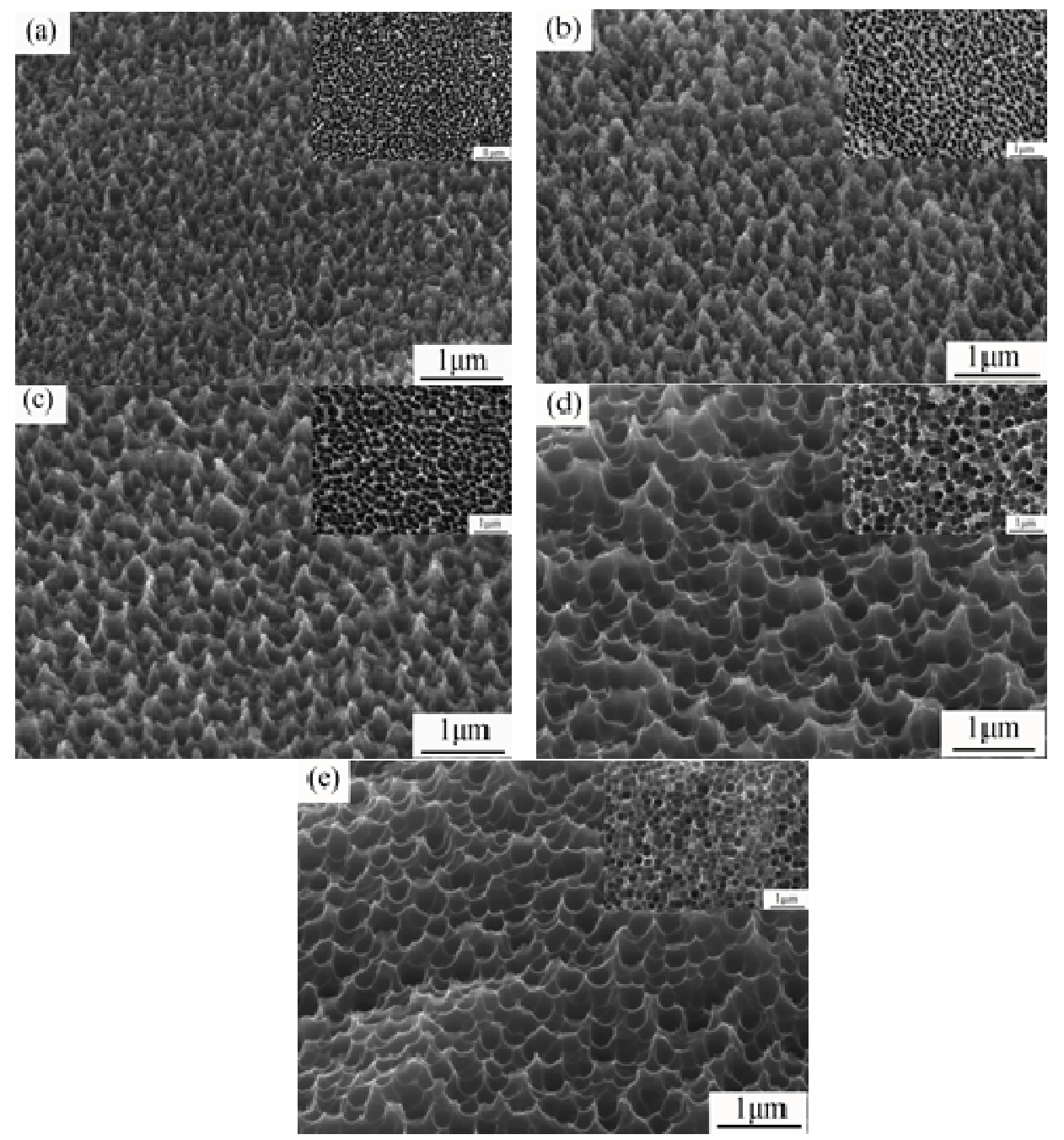

Fig. 3 The microstructure of the black silicon varying with the etching time (a) 10s; (b) 20s; (c) 30s; (d) 40s; (e) 50s 


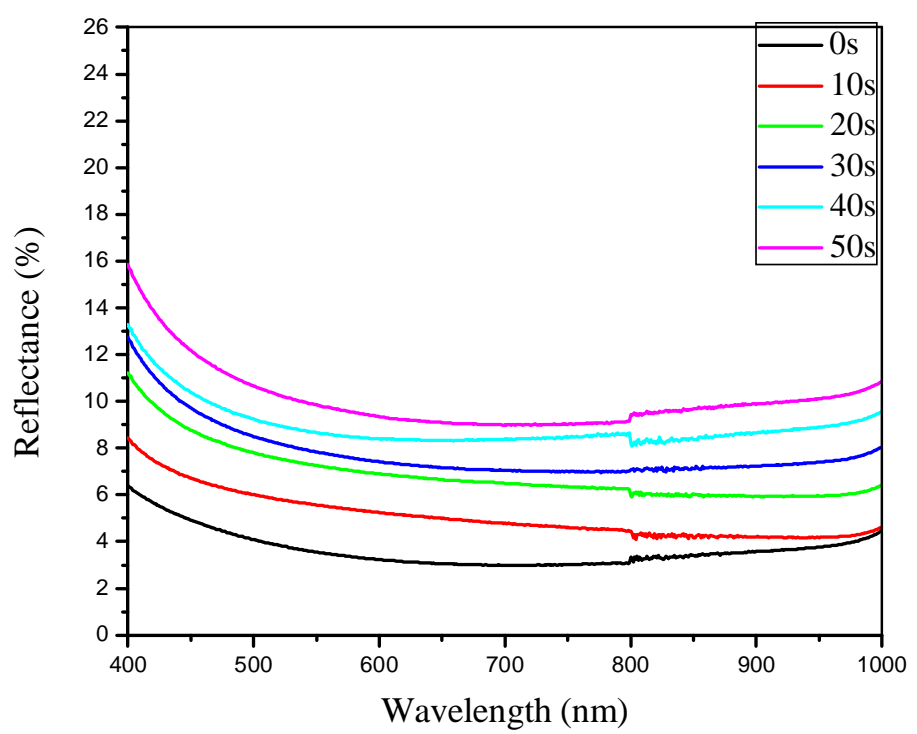

Fig. 4 The reflectance of the black silicon varying with the etching time

\section{Effect of the acid treatment on the black silicon solar cell}

Table 1 shows the influence of etching time on the properties of the black silicon solar cells. It can be seen that the efficiency and the open circuit voltage of the black silicon solar cells increase when the etching time does not exceed 20s. The increase of the efficiency can be attributed to the damage removal and the decrease of the surface defects and dangling bonds. Acid etching will optimize the microstructure of the black silicon, resulting in the improvement of the passivation which can increase the open circuit voltage. However, the efficiency of the black silicon will decrease with the etching time when the etching time exceeds 20 s. This is due to that the surface reflectance will increase when the etching time is too long.

Table 1 The influence of acid etching time on the black silicon solar cell properties

\begin{tabular}{ccccc}
\hline $\begin{array}{l}\text { Etching time } \\
(\mathrm{s})\end{array}$ & $\mathrm{U}_{\mathrm{oc}}(\mathrm{V})$ & $\mathrm{I}_{\mathrm{sc}}(\mathrm{A})$ & $\mathrm{FF}(\%)$ & $\mathrm{E}_{\mathrm{ff}}(\%)$ \\
\hline $0 \mathrm{~s}$ & 0.605 & 8.02 & 77.0 & 15.36 \\
$10 \mathrm{~s}$ & 0.611 & 8.31 & 76.6 & 15.98 \\
$20 \mathrm{~s}$ & 0.617 & 8.42 & 76.6 & 16.36 \\
$30 \mathrm{~s}$ & 0.618 & 8.37 & 75.8 & 16.13 \\
$40 \mathrm{~s}$ & 0.615 & 8.40 & 74.6 & 15.85 \\
$50 \mathrm{~s}$ & 0.615 & 8.35 & 73.8 & 15.58 \\
\hline
\end{tabular}

The reverse saturation current is a measure of the "leakage" of carriers across the p-n junction in reverse bias. This leakage is a result of carrier recombination in the neutral regions on either side of the junction. If one assumes infinite shunt resistance, the relationship between the reverse saturation current and the I-V characteristics follows the equation(1):

$$
v_{C}={ }_{q}^{k T} \ln \left(\begin{array}{c}
I_{S C} \\
I_{0}
\end{array}+1\right)
$$


where Voc is the open circuit voltage, Isc is the short circuit current and I0 is the reverse saturation current. Based this equation and I-V characteristics, the reverse saturation current of the black silicon solar cells can be obtained. The relationship of the reverse saturation current and the acid etching time is presented in figure 5. With increasing the etching time, the reverse saturation current decreases, indicating that the surface recombination has been reduced and surface damage layer has been removed. The reverse saturation current decreases obviously when the etching time is shorter than 20 s and the reverse saturation current does not change significantly after 20 s.

Figure 6 shows the internal quantum efficiency (IQE) of the black silicon solar cells varying with the etching time. With increasing the etching time, the IQE data of black silicon cell increase obviously at short wavelengths. As is known to all, high-energy (short wavelengths) light is absorbed very close to the surface, therefore, considerable recombination at the front surface will affect the IQE at short wavelengths. The IQE at short wavelengths is related with the response of the front surface of the cell. Therefore, the IQE of black silicon solar cells increases at short wavelengths due to damage layer removal by acid etching.

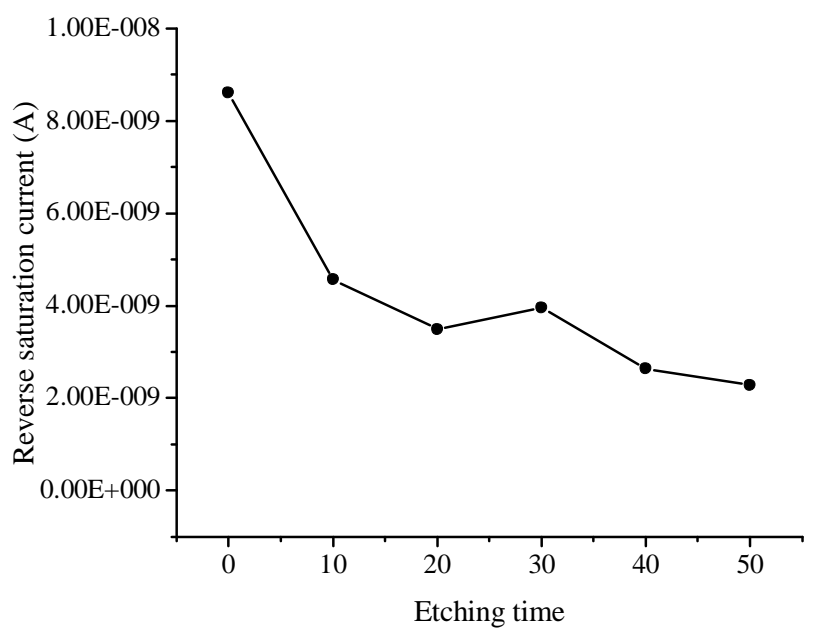

Fig. 5 The reverse saturation current of the black silicon solar cells varying with etching time

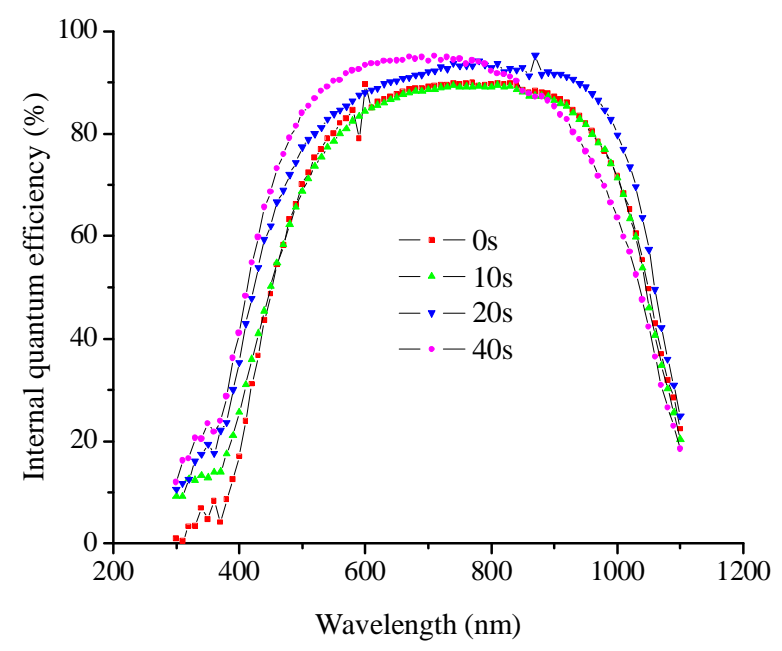

Fig. 6 The Internal quantum efficiency of the black silicon solar cells varying with etching time 


\section{Conclusions}

Black silicon produced by plasma immersion ion implantation has been treated by $\mathrm{HF} / \mathrm{HNO} 3$ mixture solutions to increase the efficiency of the black silicon solar cells. Honeycomb structure can be obtained by HNO3-rich HF-HNO3 solution treatment. With increasing the treatment time, the structure of the black silicon becomes shallow and the reflectance will increase. After etching $20 \mathrm{~s}$ in the $\mathrm{HF} / \mathrm{HNO} 3 / \mathrm{H} 2 \mathrm{O}$ solution with a ratio of $1 / 25 / 18$, the efficiency of the black silicon solar cell can be increased by $1 \%$. The improved efficiency can be attributed to structural optimization and damage removal by acid treatment.

\section{Acknowledgements}

This work was financially supported by the National Natural Science Foundation of China (Grant No. 61274059, 61106060) and Taishan Technological Leading Talent Program.

\section{References}

[1] B. Gonzalez-Diaz, R. Guerrero-Lemus, B. Diaz-Herrera, et al.: Mater. Sci. Eng., B 159-160 (2009), pp. 295-298.

[2] L. L. Ma, Y. C. Zhou, N, Jiang: Applied physics letters. 2006, 88: 171907.

[3] H. V. Jansen, M. J. de Boer, K. Ma, et al.: Journal of micromechanics and microengineering. 2010, 20: 075027.

[4] J. S. Yoo, I. O. Parm, U. Gangopadhyay, et al.: Solar energy materials and solar cells. 2006, 90: 3085-3093.

[5] G. Kumaravelu, M. M. Alkaisi, A. Bittar. Surface texturing for silicon solar cells using reactive ion etching technique. Photovoltaic Specialists Conference. Louisiana. 2002. IEEE Conference Publications. Louisiana, 2002, 258-261.

[6] J. Yoo, G. Yu, J. Yi: Materials science and engineering: B. 2009, 159-160: 333-337.

[7] M. Halbwax, T. Sarnet, Ph. Delaporte, et al.: Thin solid films. 2008, 516: 6791-6795.

[8] C. Wu, C. H. Crouch, L. Zhao, et al., Applies Physicals Letters. 2001, 78: 1850.

[9] S. Koynov, M. S. Brandt, M. Stutzmann: Physica status solidi-Rapid research letters. 2007, 1: 53-55.

[10] S. Koynov, M. S. Brandt, M. Stutzmann: Applied physics letters. 2006, 88: 203107.

[11] H. Yuan, V. E. Yost, M. R. Page, et al.: Applied physics letters. 2009, 95: 123501.

[12] Y. Xia, B. Liu, J. Liu, et al.: Solar Energy. 2011, 85: 1574-1578.

[13] Y. Xia, B. Liu, S. Zhong, and B. Chao: Journal of Electron Spectroscopy Related Phenomena. 2012, 184: 589-592.

[14] B. Liu, S. Zhong, J. Liu, et al.: International journal of photoenergy, Volume 2012: doi:10.1155/2012/971093.

[15] F. Shimura, Semiconductor Silicon Crystal Technology, Academic Press, Inc., San Diego, 1989, 184-186.

[16] S. R. Wenham, M. A. Green, M. E. Watt, et al., Applied photovoltaics. second edition. London, Earthscan, 2007, 53-54. 